\title{
Effect of Substrate Composition and Heat Pretreatment on Vegetative Growth of Agaricus brasiliensis S. Wasser et al. and A. subrufescens Peck
}

\author{
Omoanghe S. Isikhuemhen E C. Ivey \\ Mushroom Biology and Fungal Biotechnology Laboratory, School of Agriculture and \\ Environmental Sciences, North Carolina Agricultural and Technical State University, \\ Greensboro, NC 27411, USA
}

Approximately 670 million broiler chickens and 43 million turkeys are produced in North Carolina annually, generating wastes that are causing environmental problems and concerns among residents. These waste products can be used as substrate for growing specialty mushrooms. Mushroom farming can enable farmers to obtain additional crops (mushrooms), while bioconverting the agricultural wastes to more environment friendly products such as organic fertilizers. Agaricus brasiliensis and $A$. subrufescens are culinary-medicinal mushrooms currently produced in the US. These mushrooms can be alternative crops generating high income in the place of tobacco for the North Carolina farmers.

Various combinations of sawdust, poultry litter, and cotton waste were made and loaded in $3 \times 12$ $\mathrm{cm}$ glass test tubes. The tubes containing substrate were sterilized at $121^{\circ} \mathrm{C}$ for 1,2 , or 3 hours. A set of 8 tubes per substrate combination was inoculated with $A$. brasiliensis or $A$. subrufescens. Similar substrate combinations were pasteurized at $90^{\circ} \mathrm{C}$ for 48 hours and then inoculated.

Growth rates of each substrate combination/heat treatment were determined, based on the time for achieving total substrate colonization in each tube. To determine the effect of nutrient supplementation on mycelia growth and development, the substrate combination/heat treatment that performed best in the above treatments was supplemented with wheat bran or millet or combinations of both at 10, 15, and $20 \%$ proportions. Non-supplemented substrate served as a control.

Substrate combinations containing cotton waste did not show significant growth and development in either $A$. brasiliensis or $A$. subrufescens. Sterilization was ineffective at suppressing contaminants in any substrate combination that had poultry liter. Pasteurization of poultry litter containing substrates at $90^{\circ} \mathrm{C}$ for 48 hours was sufficient to suppress contaminant. Poultry litter and sawdust in a 1:1 ratio proved to be the best substrate for supporting mycelia growth, where total colonization took an average of 15 days, reaching primordial formation in 60 days. Both fungi responded well to nutrient supplementation, showing an average of $81.5 \%$ increase in mycelia growth over the control. In both fungi tested, there was consistent increase in colonization rate as supplementation increased; however, incidence of contaminants increased with the increase of supplementation.

Further work on optimization of substrate pretreatment and environmental conditions that best support the development of primordial through mature fruit body formation in both fungi are currently being researched. 